\section{Association of pediatric dental caries with passive smoking}

Aligne CA, Moss ME et al. JAMA 2003; 289: 1258-1264

Raised serum cotinine was associated with more caries.

There are good biological reasons why tobacco smoke increases periodontitis, but it is less clear whether it can affect caries, though both smoking and caries are commoner in the economicallydeprived. This study used data from a US sample of 3531 children who had dental examinations and concurrent serum cotinine measurements. Cotinine is a marker of environmental tobacco smoke (ETS) inhalation. Children aged 12+yrs, or with more than $10 \mathrm{ng} / \mathrm{ml}$ cotinine in their serum, had been excluded to avoid possible young active smokers.

Logistic regression analysis was used to control for various socio-economic variables. There were significant but rather low odds ratios for ETS exposure and decayed and filled surfaces in deciduous teeth (1.8 and 1.4 respectively), but not in permanent teeth (1.2 and 0.9). However, the ETS dose relationship with caries was not fully consistent. The authors were not able to exclude a common behavioural factor in relation to smoking and caries, because data on dental hygiene and dietary habits were not collected.

\section{CONSERVATIVE DENTAL SURGERY}

Conventional versus resin-modified glassionomer cement for Class II restorations in primary molars. A 3-year clinical study.

Hübel S, Mejàre L Int J Paediatr Dent 2003; 13: 2-8

Resin-modification proved clinically more successful.

Conventional glass-ionomer cements (GICs) have proven less satisfactory than amalgam as primary Class II restorations, but resin-modified GICs have shown more promising results in cohort studies. In this study, 40 children aged 4-7 yrs received 62 conventional GIC and 53 resin-modified GIC restorations on a split mouth basis. All restorations were placed under LA without rubber dam.

After median follow-up of 36 months, 96\% of resin modified (RM) GICs were successful, and 82\% of conventional (C) GICs. Failure incidence of RMGICs was 1.3 per 100 restored tooth-yrs, and of CGICs, 6.2. The authors considered RMGICs to be clinically advantageous.

\section{DENTAL PUBLIC HEALTH}

\section{Influences of social support on the oral} health of older people in Britain

\section{McGrath C, Bedi R J Oral Rehabil 2002; 29: 918-922}

Social support is a factor influencing the oral health behaviour of older people in UK.

From a random sample of UK residents, 3739 (69\%) participated in a statistical survey. This paper reports on 876 who were aged 65+ yrs, and who were visited and interviewed in their homes. About half were aged 75+ yrs, 40\% were male, half were of higher socioeconomic class (I, II and IIINM), and half were living alone. One quarter had 20+ teeth, 43\% wore complete dentures, 32\% wore partial dentures, 56\% had seen a dentist in the past year, and 11\% went for emergency treatment at the last visit.

Subjects living alone were significantly less likely to have gone to the dentist within the past year, and more likely to have gone as an emergency. Regression analysis showed that pain-motivated attenders were less likely to have a supportive social network (ie to be living with family or friends), and more likely to have dentures. Subjects wearing complete dentures were also less likely to have a supportive social network.

\section{ENDODONTICS}

\section{Clinical investigation of second} mesiobuccal canals in endodontically treated and retreated maxillary molars

Wolcott J, Ishley D et al. J Endodon 2002; 28: 477-479

During retreatment, a second canal was found in 2/3 of first molars, and nearly $1 / 2$ of second molars.

This study examined 1873 maxillary first and second molars consecutively root treated over a 2 yr period by 5 endodontists, using $3.5 \times$ or greater magnification and fibre-optic illumination.

In 929 initially treated first molars, 59\% were found to have a second mesio-buccal canal, and in 264 which were retreated, 67\% were found to have one $(P=0.01)$. The respective figures for 618 initially treated and 62 retreated second molars were 35\% and 44\% (NS: this is probably accounted for by low sample size).

When a combined analysis (Mantel Haenszel test) was performed, the finding of a second mesio-buccal canal was significantly associated with retreatment. Failure to find a second canal is a contributing factor to failure of root treatment in these teeth. 